\title{
Magnetoelectric torque and edge currents in spin-orbit coupled graphene nanoribbons
}

\author{
Matheus S. M. de Sousa $\odot,{ }^{1}$ Manfred Sigrist ${ }^{2}$ and Wei Chen $\odot^{1}$ \\ ${ }^{1}$ Department of Physics, PUC-Rio, 22451-900 Rio de Janeiro, Brazil \\ ${ }^{2}$ Institute for Theoretical Physics, ETH Zurich, 8093 Zurich, Switzerland
}

(Received 11 March 2021; accepted 2 June 2021; published 6 July 2021)

\begin{abstract}
For graphene nanoribbons with Rashba spin-orbit coupling, the peculiar magnetic response due to the presence of a magnetization and geometric confinement are analyzed within a tight-binding model. We observe a sizable transverse susceptibility that can be considered as a gate-voltage-induced magnetoelectric torque without the need of a bias voltage, with different directions for zigzag and armchair ribbons. The local torque generates noncollinear spin polarization between the two edges and/or along the ribbon, and the net torque averages to zero if the magnetization is homogeneous. Nevertheless, a nonzero net torque can appear in partially magnetized nanoribbons or in nanoflakes of irregular shapes. The equilibrium spin current produced by the spin-orbit coupling also appears in nanoribbons, but the component flowing in the direction of confinement is strongly suppressed. Even without the magnetization, an out-of-plane polarized helical edge spin current is produced, resembling that in the quantum spin Hall effect. Moreover, a magnetization pointing perpendicular to the edge produces a laminar flow of edge charge currents, whose flow direction is symmetric (nonchiral) or antisymmetric (chiral) between the two edges, depending on whether the magnetization points in-plane or out-of-plane.
\end{abstract}

DOI: 10.1103/PhysRevResearch.3.033021

\section{INTRODUCTION}

The celebrated Rashba spin-orbit coupling (RSOC) has a strong impact on the physical properties of two-dimensional (2D) metals with parabolic bands [1,2], especially their magnetic response and charge to spin interconversion. A number of these features originate from the RSOC-induced spinmomentum locking, such as the Edelstein effect that causes a bias-voltage-induced nonequilibrium in-plane spin polarization [3]. In magnetized 2D systems, this effect can be exploited to induce magnetization dynamics known as the spin-orbit torque [4-9], whose feasibility has been demonstrated extensively in experiments [10-12]. In addition to these properties, RSOC also modifies the equilibrium properties of 2D metals, most notably causing an in-plane polarized spin current flowing throughout the system [13-15].

Besides these effects occurring in infinitely extended 2D metals or electron gases (2DEGs), RSOC also causes peculiar effects at the boundary of geometrically confined mesoscopic $2 \mathrm{D}$ systems. A notable example is the generation of outof-plane polarized equilibrium edge spin currents $[16,17]$. Furthermore, in-plane spin polarization perpendicular to the edge can induce a persistent charge current decaying and oscillating in sign away from the edge [18]. This behavior is very similar to that found in other spin-momentum locking systems in proximity to a magnet, such as for topological

Published by the American Physical Society under the terms of the Creative Commons Attribution 4.0 International license. Further distribution of this work must maintain attribution to the author(s) and the published article's title, journal citation, and DOI. insulator/ferromagnetic metal junctions (TI/FMMs) [19,20]. The tunability of RSOC by a gate voltage may provide a means to control such phenomena and engineer dedicated devices.

In this paper, we use a tight-binding model to explore the spin torque and equilibrium currents caused by RSOC and geometric confinement in graphene nanoribbons. Numerous peculiarities make graphene nanoribbons a particularly intriguing system to study: (1) the linear dispersion near the two Dirac points that causes a unique spin-momentum locking profile [21-26], (2) the emergence of zero-energy edge states in zigzag-terminated ribbons [27-34], (3) the opening of an energy gap depending on the ribbon width for armchair ribbons [35-40], and (4) the pronounced magnetic response at some sample edges [41-43]. Moreover, it is important to note that an enhanced and gate-tunable spin-orbit coupling (SOC) has been demonstrated in graphene/transition-metal dichalcogenide (TMD) heterostructures [44-50]. In graphene/yttrium iron garnet (YIG) [51-54] and graphene/Co [55] heterostructures, both SOC and ferromagnetism are induced, which might be used as the stage for the phenomena we are going to discuss. Finally, the RSOC and magnetization are also known to cause quantum spin Hall (QSHE) and quantum anomalous Hall effects (QAHE) in graphene [56-58].

We first elaborate on the feature that, in contrast to the usual longitudinal in-plane susceptibility in an infinitely large graphene, a transverse in-plane susceptibility develops in nanoribbons due to the RSOC and geometric confinement. The controllability of the RSOC suggests that this transverse susceptibility serves as a gate-voltage-induced magnetoelectric torque on the magnetization without the need of a bias voltage, contrary to the current-induced spin-orbit torque [55,59-63]. While this torque averages to zero for a 
homogeneously spin-polarized system, well-designed local variations in the magnetization can yield a net torque signal available to practical purpose. We further investigate the pattern of equilibrium spin currents in nanoribbons, especially concerning the symmetry of flow directions between the two edges, e.g., distinguished helical and nonhelical structure. Charge currents induced by spin polarization exist in nanoribbons too, whose variability on the direction of the magnetization will be a point of attention.

We start by introducing the lattice model for graphene with RSOC and spin magnetization, and then calculate the persistent spin current to show how the spin-momentum locking is modified by the presence of the magnetization in Sec. II. In Sec. III, we use a two-site toy model to demonstrate analytically the existence of a transverse susceptibility due to geometric confinement, which is then transferred to the discussion of both zigzag and armchair ribbons. In addition, we characterize the patterns and chiralities of persistent charge and spin currents, and also use a specific example to demonstrate that these phenomena also survive in graphene nanoflakes. Section IV summarizes our results.

\section{EXTENDED TIGHT-BINDING MODEL OF GRAPHENE}

In order to emphasize the impact of geometric confinement, we start by addressing the spintronics-related effects due to RSOC for an infinite graphene sheet, before discussing the same physics for graphene ribbons and nanoflakes. As indicated in Fig. 1(a), three lattice vectors (in units of bond length $a=1$ ) characterize the honeycomb lattice,

$$
\boldsymbol{\delta}_{1}=\left(\frac{1}{2}, \frac{\sqrt{3}}{2}\right), \quad \boldsymbol{\delta}_{2}=\left(\frac{1}{2},-\frac{\sqrt{3}}{2}\right), \quad \boldsymbol{\delta}_{3}=(-1,0),
$$

connecting neighboring lattice sites belonging to the two different sublattices, $A$ and $B$. We now formulate the following tight-binding model that incorporates both RSOC and spin polarization [64-68]:

$$
\begin{aligned}
H= & -t \sum_{\langle i j\rangle, \sigma} c_{i \sigma}^{\dagger} c_{j \sigma}+J_{\mathrm{ex}} \sum_{i, \alpha, \beta} \mathbf{S} \cdot c_{i \alpha}^{\dagger} \boldsymbol{\sigma}_{\alpha \beta} c_{i \beta} \\
& +i \lambda_{R} \sum_{\langle i j\rangle, \alpha, \beta} c_{i \alpha}^{\dagger}\left(\boldsymbol{\sigma}_{\alpha \beta} \times \mathbf{d}_{i j}\right)^{z} c_{j \beta}-\mu \sum_{i \sigma} c_{i, \sigma}^{\dagger} c_{i \sigma} .
\end{aligned}
$$

Here $c_{i \sigma}^{\dagger}\left(c_{i \sigma}\right)$ creates (annihilates) an electron of spin $\sigma$ on the lattice site $i$, where sums with $\langle i j\rangle$ run over nearestneighbor lattice sites. The hopping matrix element is $-t$, $\lambda_{R}$ is the RSOC coupling constant, $\sigma=\left(\sigma^{x}, \sigma^{y}, \sigma^{z}\right)$ are the spin Pauli matrices, $\mathbf{d}_{i j}$ is the vector connecting the site $i$ to $j, J_{\mathrm{ex}}$ is the exchange coupling between the magnetization $\mathbf{S}=S(\sin \theta \cos \phi, \sin \theta \sin \phi, \cos \theta)$ and the spin, and $\mu$ is the chemical potential. The bipartite structure of our system allows us to use a sublattice formulation with $I=\{A, B\}$, where we define the basis $\psi=(A \uparrow, B \uparrow, A \downarrow, B \downarrow)$, and the electron operators in Eq. (2) can be split into $c_{i \sigma} \rightarrow\left\{c_{A i \sigma}, c_{B i \sigma}\right\}$, where $i$ now denotes the position of each unit cell containing an $A$ and $B$ lattice site. After Fourier transformation $c_{I i \sigma}=$ $\sum_{\mathbf{k}} e^{i \mathbf{k} \cdot \mathbf{r}_{i}} c_{I \mathbf{k} \sigma}$, the Hamiltonian $H=\sum_{\mathbf{k I J} \alpha \beta} c_{I \mathbf{k} \alpha}^{\dagger} H_{I \alpha J \beta}(\mathbf{k}) c_{J \mathbf{k} \beta}$

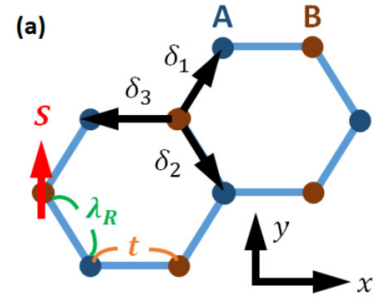

(c)

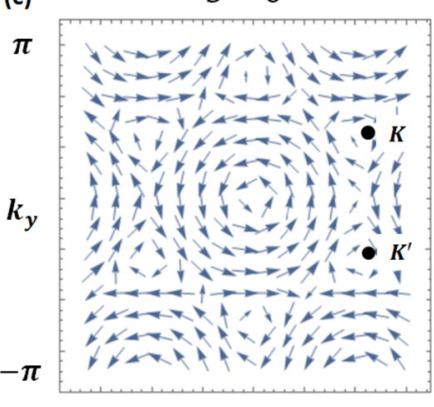

$S \| y$

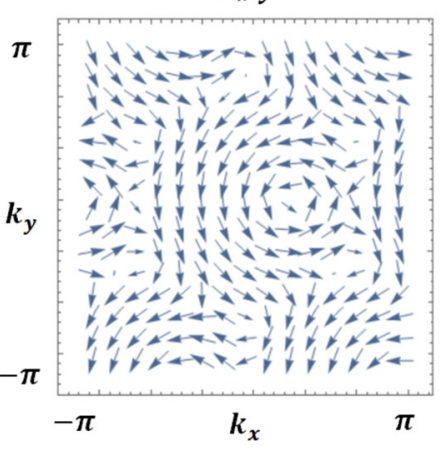

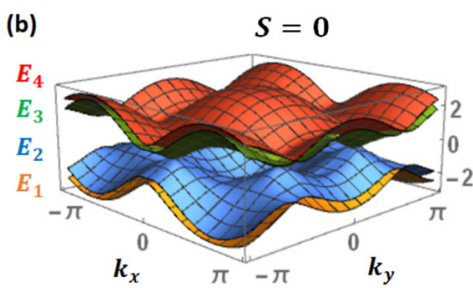

$S \| x$

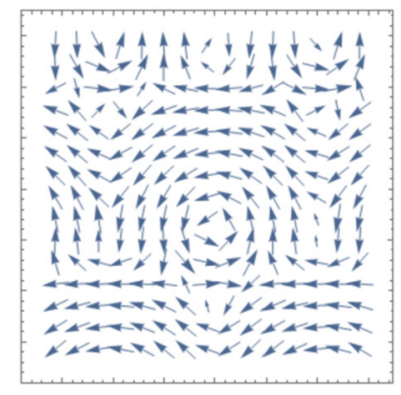

$S \| Z$

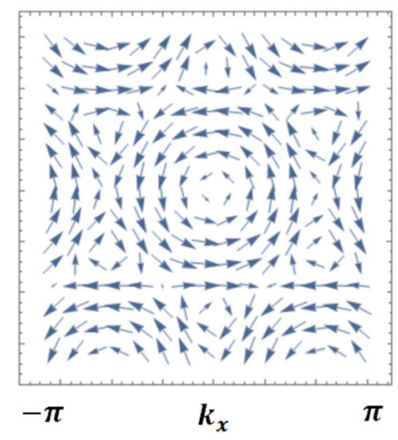

FIG. 1. (a) The definition of coordinates in our honeycomb lattice. (b) The resulting band structure in the presence of RSOC without magnetization $\mathbf{S}=\mathbf{0}$. (c) The spin-momentum locking profile of the lowest eigenstate $\left|u_{\mathbf{k} 1}\right\rangle$ in the absence $\mathbf{S}=\mathbf{0}$ and presence $\mathbf{S} \|\{\hat{\mathbf{x}}, \hat{\mathbf{y}}, \hat{\mathbf{z}}\}$ of the magnetization.

defines the matrix

$$
\begin{aligned}
& H_{I \alpha J \beta}(\mathbf{k}) \\
& \quad=\left(\begin{array}{cccc}
J_{\mathrm{ex}} S^{z} & t Z^{*} & J_{\mathrm{ex}} S_{\perp} e^{-i \phi} & \lambda_{R} Y^{*} \\
t Z & J_{\mathrm{ex}} S^{z} & \lambda_{R} X^{*} & J_{\mathrm{ex}} S_{\perp} e^{-i \phi} \\
J_{\mathrm{ex}} S_{\perp} e^{i \phi} & \lambda_{R} X & -J_{\mathrm{ex}} S^{z} & t Z^{*} \\
\lambda_{R} Y & J_{\mathrm{ex}} S_{\perp} e^{i \phi} & t Z & -J_{\mathrm{ex}} S^{z}
\end{array}\right),
\end{aligned}
$$

with

$$
\begin{aligned}
& Z \equiv e_{1}+e_{2}+e_{3}, \\
& X \equiv \frac{-1-i \sqrt{3}}{2} e_{1}^{*}+\frac{-1+i \sqrt{3}}{2} e_{2}^{*}+e_{3}^{*}, \\
& Y \equiv \frac{1+i \sqrt{3}}{2} e_{1}+\frac{1-i \sqrt{3}}{2} e_{2}-e_{3},
\end{aligned}
$$

where we have defined $e_{a}=e^{i \mathbf{k} \cdot \delta_{a}}$ and $S_{\perp}=$ $\sqrt{\left(S^{x}\right)^{2}+\left(S^{y}\right)^{2}}=S \sin \theta \quad(\theta \in[0, \pi])$. In the following we choose the parameters

$$
J_{\mathrm{ex}}=0.2, \quad \lambda_{R}=0.2, \quad \mu=0.5, \quad k_{B} T=0.03,
$$


with $t=1$ as the energy unit, such that the magnetic response is more pronounced, but we emphasize that their patterns are fairly robust against variation of these parameters.

Diagonalizing the Hamiltonian yields two filled bands $E_{\mathbf{k} 1}<E_{\mathbf{k} 2}<0$ and two empty bands $0<E_{\mathbf{k} 3}<E_{\mathbf{k} 4}$, with the corresponding four eigenstates $\left\{\left|u_{\mathbf{k} 1}\right\rangle,\left|u_{\mathbf{k} 2}\right\rangle,\left|u_{\mathbf{k} 3}\right\rangle,\left|u_{\mathbf{k} 4}\right\rangle\right\}$. These four bands are shown in Fig. 1(b) for zero magnetization. In this case the spin expectation value $\langle\sigma\rangle_{\mathbf{k} \eta}=$ $\left\langle u_{\mathbf{k} \eta}|\sigma| u_{\mathbf{k} \eta}\right\rangle$ for each eigenstate has only in-plane components $\left\{\left\langle\sigma^{x}\right\rangle_{\mathbf{k} \eta},\left\langle\sigma^{y}\right\rangle_{\mathbf{k} \eta}\right\}$, as displayed in the vector plots in Fig. 1(c) for $S=0$, where we also see $\langle\boldsymbol{\sigma}\rangle_{\mathbf{k} 1}=-\langle\boldsymbol{\sigma}\rangle_{\mathbf{k} 2}$. We see that the spin texture forms a vortex around each Dirac point, $\mathbf{K}=(2 \pi / 3,2 \pi / 3 \sqrt{3})$ and $\mathbf{K}^{\prime}=(2 \pi / 3,-2 \pi / 3 \sqrt{3})$ with the same vorticity, but opposite vorticity appears for the vortex around the origin $\mathbf{k}=(0,0)$. This spin pattern represents the spin-momentum locking due to the RSOC.

As common in 2D Rashba metals, the presence of a magnetization $\mathbf{S}$ is expected to distort the profile of the spin pattern [5,7], as demonstrated for magnetizations along the three principle directions $\mathbf{S} \|\{\hat{\mathbf{x}}, \hat{\mathbf{y}}, \hat{\mathbf{z}}\}$ in Fig. 1(c), which shows the planar components of $\langle\boldsymbol{\sigma}\rangle_{\mathbf{k} 1}$ only. For $\mathbf{S} \| \hat{\mathbf{x}}$, the $\langle\boldsymbol{\sigma}\rangle_{\mathbf{k} 1}$ becomes asymmetric between $+k_{y}$ and $-k_{y}$, which also causes an asymmetry in the dispersion in this direction. Analogously, $\mathbf{S} \| \hat{\mathbf{y}}$, yields an asymmetry between $+k_{x}$ and $-k_{x}$, whereas for $\mathbf{S} \| \hat{\mathbf{z}}$, the planar components basically remain the same as that in the absence of magnetization. By calculating the spin polarization in our lattice model with a periodic boundary condition (PBC), we also verify that the spin polarization on either sublattice is always longitudinal, i.e., along the direction of magnetization.

The charge and spin current density operators are constructed from the equations of motion of the charge and spin density operator, respectively, which correspond to continuity equations $[19,20]$

$$
\begin{aligned}
\dot{n}_{i} & =\frac{i}{\hbar}\left[H, n_{i}\right]=\frac{i}{\hbar}\left[H_{t}+H_{R}, n_{i}\right] \\
& =-\nabla \cdot \mathbf{J}_{i}^{0}=-\frac{1}{a} \sum_{\eta} J_{i, i+\eta}^{0}, \\
\dot{m}_{i}^{a} & =\frac{i}{\hbar}\left[H, m_{i}^{a}\right]=\frac{i}{\hbar}\left[H_{t}+H_{R}, m_{i}^{a}\right]+\frac{i}{\hbar}\left[H_{J}, m_{i}^{a}\right] \\
& =-\nabla \cdot \mathbf{J}_{i}^{a}+\tau_{i}^{a} \\
& =-\frac{1}{a} \sum_{\eta} J_{i, i+\eta}^{a}+\frac{2 J_{\mathrm{ex}}}{\hbar}\left(\mathbf{S} \times c_{i \alpha} \boldsymbol{\sigma}_{\alpha \beta} c_{i \beta}\right)^{a},
\end{aligned}
$$

where $\boldsymbol{\eta}=\left(-\boldsymbol{\delta}_{1},-\boldsymbol{\delta}_{2},-\boldsymbol{\delta}_{3}\right)$ if $i \in A$, and $\boldsymbol{\eta}=\left(\boldsymbol{\delta}_{1}, \boldsymbol{\delta}_{2}, \boldsymbol{\delta}_{3}\right)$ if $i \in B$. Moreover, $\tau_{i}^{a}=\frac{2 J_{\mathrm{ex}}}{\hbar}\left(\mathbf{S} \times c_{i \alpha} \boldsymbol{\sigma}_{\alpha \beta} c_{i \beta}\right)^{a}$ denotes the spin torque term as originating from the usual Landau-Lifshitz dynamics. The calculation of the commutators in Eq. (6) is detailed in the Appendix.

\section{GRAPHENE NANORIBBONS WITH MAGNETIZATION AND RASHBA SPIN-ORBIT COUPLING}

\section{A. Two-site toy model}

For deeper insight into the impact of geometrical confinement on properties of graphene with RSOC and magnetization, we first present an exactly solvable two-site toy model to demonstrate the feature of the transverse spin susceptibility and the persistent spin current. Similar models have been proposed to explain the microscopic mechanism of noncollinear magnetic order and equilibrium spin currents [69-71], while we will put emphasis on the spin torque here. The two-site model Hamiltonian reads

$$
\begin{aligned}
H= & \sum_{\sigma} t\left(c_{A \sigma}^{\dagger} c_{B \sigma}+c_{B \sigma}^{\dagger} c_{A \sigma}\right)+J_{\mathrm{ex}} \sum_{i=A, B} \mathbf{S}_{i} \cdot c_{i \alpha}^{\dagger} \boldsymbol{\sigma}_{\alpha \beta} c_{i \beta} \\
& +i \lambda_{R} \sum_{i j} c_{i \alpha}^{\dagger}\left(\boldsymbol{\sigma}_{\alpha \beta} \times \mathbf{d}_{i j}\right)^{z} c_{j \beta},
\end{aligned}
$$

where the two sites $i=\{A, B\}$ are assumed to be connected along $\mathbf{d}_{A B}=-\mathbf{d}_{B A} \| \hat{\mathbf{x}}$, and we consider the same magnetization on the two sites $\mathbf{S}_{A}=\mathbf{S}_{B}$. The two sites feature the $A$ and $B$ sublattice sites in the graphene unit cell connected along the $\hat{\mathbf{x}}$ direction, as implied in Fig. 1 . The $4 \times 4$ Hamiltonian in the basis of $\left(c_{A \uparrow}, c_{B \uparrow}, c_{A \downarrow}, c_{B \downarrow}\right)$ is

$$
H=\left(\begin{array}{cccc}
J_{\mathrm{ex}} S^{z} & t & J_{\mathrm{ex}} S_{\perp} e^{-i \phi} & -\lambda_{R} \\
t & J_{\mathrm{ex}} S^{z} & \lambda_{R} & J_{\mathrm{ex}} S_{\perp} e^{-i \phi} \\
J_{\mathrm{ex}} S_{\perp} e^{i \phi} & \lambda_{R} & -J_{\mathrm{ex}} S^{z} & t \\
-\lambda_{R} & J_{\mathrm{ex}} S_{\perp} e^{i \phi} & t & -J_{\mathrm{ex}} S^{z}
\end{array}\right),
$$

analogous to Eq. (3). Assuming $\left\{J_{\mathrm{ex}}, \lambda_{R}, t\right\}>0$ and $\left\{J_{\text {ex }}, \lambda_{R}\right\} \ll t$, for any magnetization direction there are two negative $\left(E_{1}, E_{2}\right)$ and two positive $\left(E_{3}, E_{4}\right)$ eigenenergies. Suppose the two negative eigenstates $\left|u_{1}\right\rangle$ and $\left|u_{2}\right\rangle$ are occupied. Then the $\alpha$ component spin expectation value on site $i$ is given by $\left\langle\sigma_{i}^{\alpha}\right\rangle=\left\langle u_{1}\left|\sigma_{i}^{\alpha}\right| u_{1}\right\rangle+\left\langle u_{2}\left|\sigma_{i}^{\alpha}\right| u_{2}\right\rangle$, which can be expanded in powers of $J_{\text {ex }} S$ to obtain the susceptibility along principle directions $\chi_{i}^{\alpha \beta}=\partial\left\langle\sigma_{i}^{\alpha}\right\rangle / \partial\left(J_{\text {ex }} S^{\beta}\right)$. We find

$$
\begin{aligned}
\chi_{A}^{\alpha \beta} & =\left(\begin{array}{lll}
\chi_{A}^{x x} & \chi_{A}^{x y} & \chi_{A}^{x z} \\
\chi_{A}^{y x} & \chi_{A}^{y y} & \chi_{A}^{y z} \\
\chi_{A}^{z x} & \chi_{A}^{z y} & \chi_{A}^{z z}
\end{array}\right) \\
& =\frac{1}{\left(t^{2}+\lambda_{R}^{2}\right)^{3 / 2}}\left(\begin{array}{ccc}
-\lambda_{R}^{2} & 0 & t \lambda_{R} \\
0 & 0 & 0 \\
-t \lambda_{R} & 0 & -\lambda_{R}^{2}
\end{array}\right)=\left(\chi_{B}^{\alpha \beta}\right)^{T} .
\end{aligned}
$$

Thus there is a transverse response $\left\{\chi^{x z}, \chi^{z x}\right\}$ between the $x$ and $z$ directions, and consequently a spin torque on both sites due to Landau-Lifshitz dynamics,

$$
\frac{d \mathbf{S}_{i}}{d t}=\frac{J_{\mathrm{ex}}}{\hbar}\left\langle\boldsymbol{\sigma}_{i}\right\rangle \times \mathbf{S}_{i} .
$$

However, because the transverse susceptibility is opposite on the two sites $\chi_{A}^{x z}=-\chi_{B}^{x z}$, the net torque $\sum_{i=A, B} d \mathbf{S}_{i} / d t$ vanishes. If we assume a coupling of the two magnetic moments by some exchange interaction $J_{A B} \mathbf{S}_{A} \cdot \mathbf{S}_{B}$, then the transverse spin polarization causes a canting angle $\theta_{A B} \approx$ $2 \cos ^{-1}\left(J_{\text {ex }}^{2} \lambda_{R} / 2 J_{A B} t^{2}\right)$ between them in the $x y$ plane, realizing a Dzyaloshinskii-Moriya interaction (DMI) [72,73]. Note that $\chi_{A}^{x x}$ and $\chi_{A}^{z z}$ in Eq. (9) represent the corrections to the longitudinal spin polarization due to RSOC, which must be negative, since the spins would be fully polarized $\left\langle\boldsymbol{\sigma}_{i}\right\rangle=1$ along $\mathbf{S}$ if RSOC were absent. The $\chi_{A}^{y y}=0$ means the magnetic response in the $\hat{\mathbf{y}}$ direction is unaffected.

The local charge and spin current operators in this toy model can be constructed from the same formalism used in 
Sec. II. Denoting the current operator $J_{A B}^{a}$ as the one flowing from site $A$ to $B$ and $J_{B A}^{a}$ for the opposite direction, we find that for either $\mathbf{S} \| \hat{\mathbf{x}}$ or $\mathbf{S} \| \hat{\mathbf{z}}$, the only nonzero expectation value is the spin current $\left\langle J^{y}\right\rangle$, and it satisfies

$$
\begin{aligned}
\left\langle J_{A B}^{y}\right\rangle & =-\left\langle J_{B A}^{y}\right\rangle \\
& =\frac{J_{\mathrm{ex}} S \lambda_{R}}{\sqrt{\left(t-J_{\mathrm{ex}} S\right)^{2}+\lambda_{R}^{2}}}-\frac{J_{\mathrm{ex}} S \lambda_{R}}{\sqrt{\left(t+J_{\mathrm{ex}} S\right)^{2}+\lambda_{R}^{2}}} .
\end{aligned}
$$

There is no spin current for $\mathbf{S} \| \hat{\mathbf{y}}$. For all cases the charge current is always absent, but all three components of the spin current can be nonzero, in general. Moreover, the $x$ and $z$ components of the spin currents flowing in the two directions are not negative of each other $\left\langle J_{A B}^{x, z}\right\rangle \neq-\left\langle J_{B A}^{x, z}\right\rangle$, but the continuity equation $\left\langle\dot{m}_{A}^{a}\right\rangle=\left\langle\dot{m}_{B}^{a}\right\rangle=0$ is explicitly satisfied, if the spin torque is taken into account as in Eq. (6).

\section{B. Persistent currents and spin torques in zigzag ribbons}

The results in the two-site model suggests the existence of spin torque and equilibrium spin current in geometrically confined Rashba systems, which motivates us to investigate our graphene model in Sec. II in the nanoribbon geometry. We first consider the zigzag ribbon by taking the open boundary condition (OBC) in the $\hat{\mathbf{x}}$ direction and the PBC along the $\hat{\mathbf{y}}$ direction for our lattice model in Eq. (2). There are two kinds of zigzag ribbons as far as the symmetry between the two edges is concerned, namely the mirror-symmetric and the glide-plane-symmetric ones, each invariant under the corresponding symmetry operation

$$
P_{\mathrm{zig}}^{\mathrm{mir}}=\left\{P_{x} \mid \mathbf{0}\right\}, \quad P_{\mathrm{zig}}^{\mathrm{gli}}=\left\{P_{x} \mid \sqrt{3} / 2 \hat{\mathbf{y}}\right\},
$$

using the common notation of space group operation, where $P_{x}$ denotes the mirror reflection in $\hat{\mathbf{x}}$ directions with respect to the central axis of the ribbon. We find that the symmetries of the patterns of the currents $J^{\alpha}$ and spin polarization $\sigma^{\alpha}$ in the mirror-symmetric ribbon defined with respect to $P_{\mathrm{zig}}^{\mathrm{mir}}$ are the same as those in the glide-plane-symmetric ribbon defined with respect to $P_{\mathrm{zig}}^{\mathrm{gli}}$, so we only present the former ones for simplicity. In particular, we use the zigzag ribbon of 24-site width as an example, as shown in Fig. 2. The patterns are translationally invariant along the ribbon direction $\hat{\mathbf{y}}$, and are either symmetric (labeled SY) or antisymmetric (labeled AS) under $P_{\mathrm{zig}}^{\mathrm{mir}}$, such that we only show the left half of a unit cell, with size of the arrows and disks indicating the magnitude of the currents and spin polarization, respectively. In addition, to clarify the origin of the edge charge current, for each eigenstate $\left|n_{x}, k_{y}\right\rangle$ we calculate the weight of the wave function closer to the left (LE) and right (RE) edges

$$
\begin{aligned}
& n_{n_{x}, k_{y}}^{L E}=\sum_{1 \leqslant x \leqslant N_{x} / 2}\left|\psi_{n_{x}, k_{y}}(x)\right|^{2}, \\
& n_{n_{x}, k_{y}}^{R E}=\sum_{N_{x} / 2+1 \leqslant x \leqslant N_{x}}\left|\psi_{n_{x}, k_{y}}(x)\right|^{2} .
\end{aligned}
$$

Likewise, to understand the spin current $J^{z}$ at the left edge, we calculate the spin polarization $\sigma^{z}$ at the left edge for each eigenstate,

$$
m_{n_{x}, k_{y}}^{z, L E}=\sum_{1 \leqslant x \leqslant N_{x} / 2}\left\langle\sigma_{n_{x}, k_{y}}^{z}(x)\right\rangle
$$

These quantities are represented by colors in the band structure. The results are summarized below according to different magnetization directions.

(i) $\mathbf{S}=\mathbf{0}$ : In the absence of magnetization, the nonhelical $\left\{J^{x}, J^{y}\right\}$ flowing along $\{\hat{\mathbf{y}}, \hat{\mathbf{x}}\}$ induced by RSOC are present in the zigzag ribbon, whereby $J^{y}$ is largely suppressed due to $\mathrm{OBC}$ in $\hat{\mathbf{x}}$ [74]. A helical (spin chiral) edge spin current $J^{z}$ is also produced, as hinted by the results in 2DEG [16,17,75], although in zigzag ribbons it exists even without a magnetic field and demonstrates helicity. In Fig. 2 the colored band structure clarifies the origin of $J^{z}$ : For every left edge (LE) spin up $k_{y}$ state (blue) there exists a corresponding LE spin down $-k_{y}$ state (green), yielding counterpropagating spins at the LE. This is true for all eigenstates, so the finite $J^{z}$ is not only the result of low-energy states, similar to that which has been discussed recently for the QSHE in topological insulators [76]. These features for the spin currents remain true even in the presence of a finite magnetization $\mathbf{S} \neq \mathbf{0}$.

(ii) $\mathbf{S} \| \hat{\mathbf{x}}$ : It is known that in 2DEG with RSOC, an in-plane magnetization pointing perpendicular to the edge produces an edge charge current, whose flow direction depends on the distance away from the edge [18]. S $\| \hat{\mathbf{x}}$ corresponds to this situation, in which we indeed see a charge current $J^{0}$ that is symmetric (nonchiral) between the two edges. The eigenstates are not particularly localized at either edge (the band structure is not particularly red or green), but the band structure becomes asymmetric $E\left(n_{x}, k_{y}\right)=-E\left(n_{x},-k_{y}\right)$, which can cause $J^{0}$ along the ribbon, a mechanism that has been pointed out for a superconductor/noncollinear magnet [77] and TI/FMM heterostructures [19,20]. A large transverse spin polarization $\sigma^{z}$ is induced near the edge without any bias voltage (in contrast to that produced by a bias voltage [78-85]), similar to that uncovered previously in a low-energy Dirac model [86], signaling the existence of a local torque according to the Landau-Lifshitz dynamics in Eq. (10). The torque is antisymmetric between the two edges and hence averages to zero, and is expected to cause a noncollinear order between the two edges as in the two-spin model in Sec. III A. For the parameters in Eq. (5), the largest edge currents are of the order of $\sim 0.01$, in units of et $/ \hbar \sim 10^{-4}$ A for $J_{y}^{0}$ and $\mu_{B} t / \hbar \sim 10^{15} \mu_{B} / \mathrm{s}$ for $\left\{J^{x}, J^{y}, J^{z}\right\}$, where $\mu_{B}$ is the Bohr magneton, and the largest edge spin polarizations are of the order of $\sim 0.01 \mu_{B}$.

(iii) $\mathbf{S} \| \hat{\mathbf{y}}$ : For the case of a magnetization along the ribbon, we find no charge current $J^{0}$ and no transverse spin polarization $\sigma^{x}=\sigma^{z}=0$, and hence there is no local torque. The energy spectrum is half-metallic.

(iv) $\mathbf{S} \| \hat{\mathbf{z}}$ : Interestingly, we find that an out-of-plane magnetization also produces a charge current $J^{0}$, but it is antisymmetric (chiral) between the two edges. Comparing with that in the $\mathbf{S} \| \hat{\mathbf{x}}$ case, this suggests the chirality of $J^{0}$ can be controlled by the orientation of the magnetization. This current only occurs when the chemical potential is finite $\mu \neq 0$, similar to that in the QAHE in Chern insulators [76], although our spectrum remains gapped. The band structure 

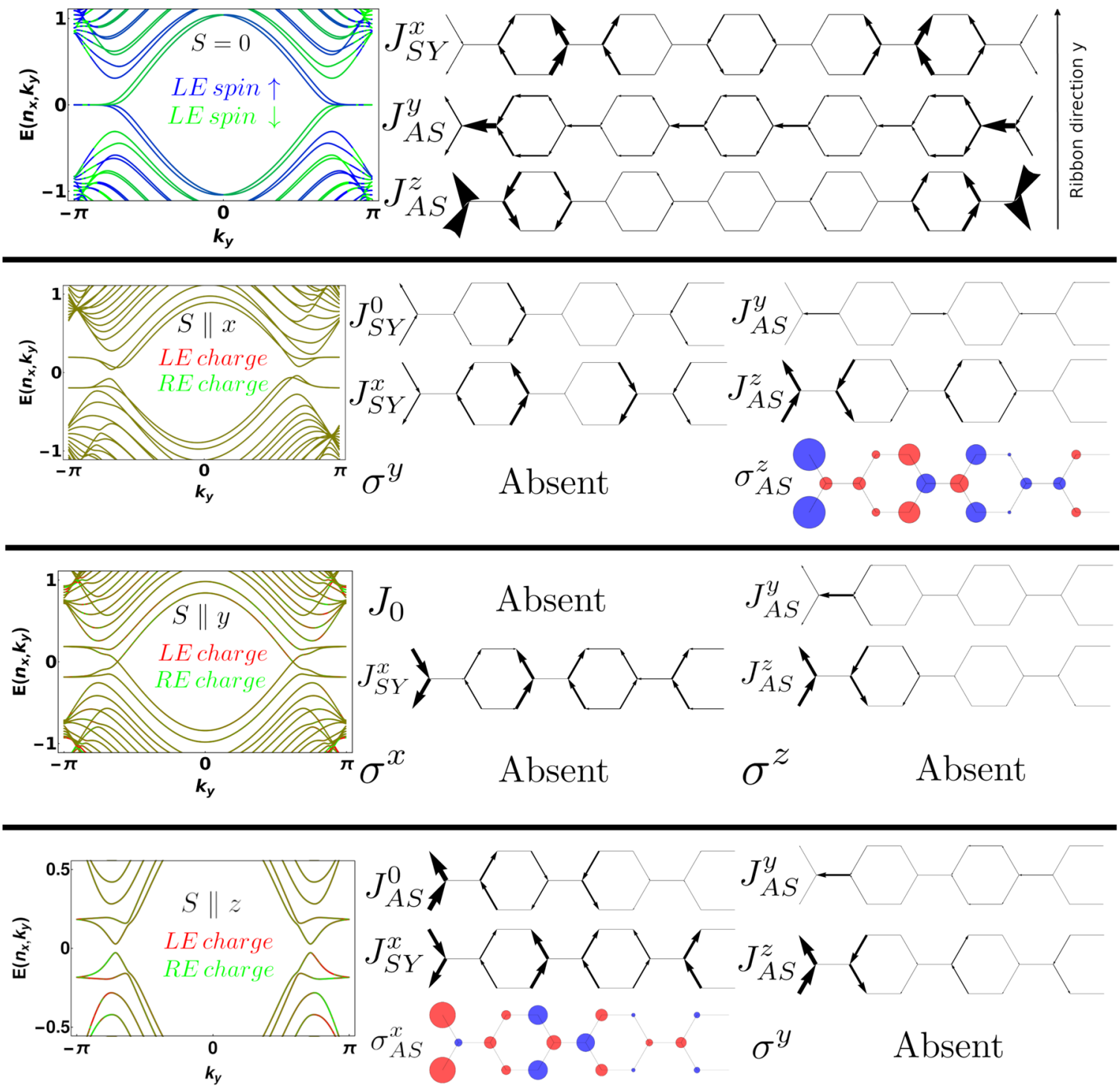

FIG. 2. The band structure $E\left(n_{x}, k_{y}\right)$ at zero chemical potential, persistent charge and spin currents $J^{\alpha}$, and transverse spin polarization $\sigma^{\alpha}$ (red positive and blue negative) in the mirror-symmetric zigzag ribbon with RSOC at different magnetization directions $\mathbf{S}$. The pattern of these quantities repeats along the ribbon direction $\hat{\mathbf{y}}$, and is either symmetric (labeled SY) or antisymmetric (labeled AS) under $P_{\mathrm{zig}}^{\mathrm{mir}}$ in Eq. (12), so we only present the left half of a unit cell, except for $\mathbf{S}=\mathbf{0}$, for which we show the entire unit cell. For the absence of magnetization case $\mathbf{S}=\mathbf{0}$, the colors in the band structure indicate the spin up and down components at the left half space (LE) for the eigenstates $\left|n_{x}, k_{y}\right\rangle$, and for $\mathbf{S} \neq \mathbf{0}$ cases they indicate the probability of the eigenstates at the left and right (RE) half space. The largest arrow in the figure has magnitude 0.015 , and the largest disk 0.011 .

is symmetric between $+k_{y}$ and $-k_{y}$ at $\mathbf{S} \| \hat{\mathbf{z}}$, but the wave function distribution is not: If the $+k_{y}$ state is mostly localized at the left edge (red), then the $-k_{y}$ state is more localized at the right edge (green), suggesting counterpropagating charge currents at the two edges. Note that some of the low-energy states are inherited from the flat band edge states of the pristine zigzag ribbon, which become dispersive and chiral under the influence of magnetization and RSOC. Finally, transverse spin polarizations $\sigma^{x}$ occur near the edges, which are opposite at the two edges and hence average to zero.

We conclude that for magnetization along principle directions $\mathbf{S} \|\{\hat{\mathbf{x}}, \hat{\mathbf{y}}, \hat{\mathbf{z}}\}$, only $\left\{\chi^{x z}, \chi^{z x}\right\}$ of the transverse susceptibility are nonzero, which then yield a local spin torque that requires no bias voltage according to Eq. (10). However, the transverse spin polarization is always antisymmetric between the two edges and hence integrates to zero $\sum_{i} d \mathbf{S}_{i} / d t=0$, indicating no net torque on a macroscopic scale. These features are similar to those in the two-site model in Sec. III A.

\section{Persistent currents and spin torques in armchair ribbons}

The armchair ribbons are simulated by imposing PBC in $\hat{\mathbf{x}}$ and $\mathrm{OBC}$ in $\hat{\mathbf{y}}$ to Eq. (2). The symmetry between the two edges distinguishes the mirror-symmetric and glideplane-symmetric armchair ribbons, with the corresponding symmetry operators

$$
P_{\mathrm{arm}}^{\mathrm{mir}}=\left\{P_{y} \mid \mathbf{0}\right\}, \quad P_{\mathrm{arm}}^{\mathrm{gli}}=\left\{P_{y} \mid 3 / 2 \hat{\mathbf{x}}\right\},
$$

where $P_{y}$ denotes the mirror operation along $\hat{\mathbf{y}}$ with respect to the central axis of the ribbon. The symmetry properties of 

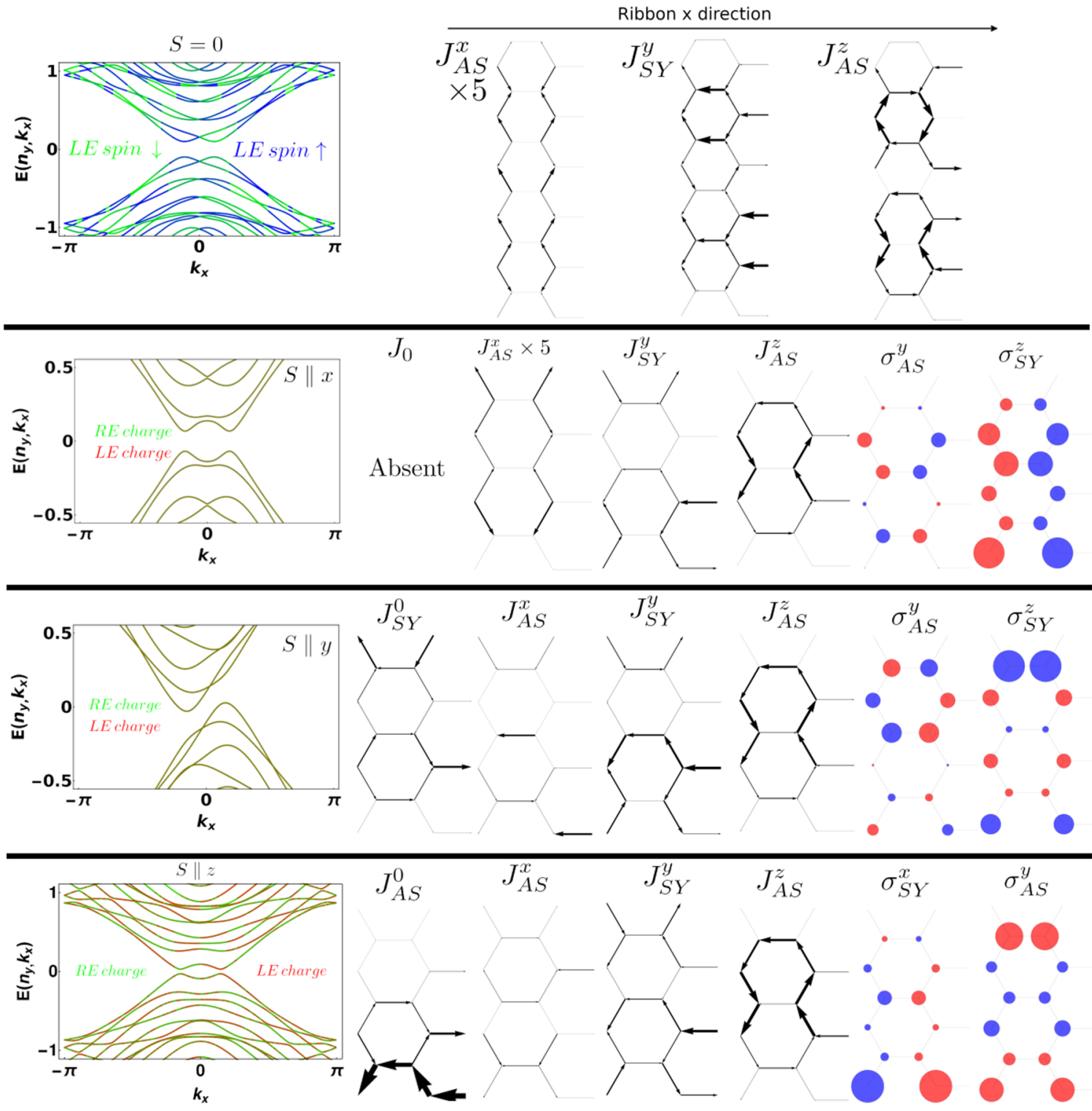

FIG. 3. Same quantities as in Fig. 2 but for glide-plane-symmetric armchair ribbon. The pattern is symmetric (labeled SY) or antisymmetric (labeled AS) as defined with respect to $P_{\mathrm{arm}}^{\mathrm{gli}}$ in Eq. (15). The largest arrow and disk in the figure are of the magnitude 0.024 and 0.011 , respectively.

the currents and spin polarization for the mirror- and glideplane-symmetric armchair ribbons, defined under $P_{\mathrm{arm}}^{\mathrm{mir}}$ and $P_{\text {arm }}^{\text {gli }}$, respectively, are identical, so we only present the glideplane-symmetric case in Fig. 3. The edge spin and charge operators are analogous to those introduced in Eqs. (13) and (14), with an exchange of coordinates $x \leftrightarrow y$. The results for different magnetization directions are summarized below.

(i) $\mathbf{S}=\mathbf{0}$ : The case $S=0$ has both the nonhelical $J^{x}$ and $J^{y}$ caused by RSOC. However, $J^{x}$ is strongly suppressed due to $\mathrm{OBC}$ in the $\hat{\mathbf{y}}$ direction. The out-of-plane polarized helical $J^{z}$ at the edge is clearly visible, and also originates from the counterpropagating spins as can be deduced from the color codes of the band structure. These features of spin currents remain valid in the $\mathbf{S} \neq \mathbf{0}$ cases below.

(ii) $\mathbf{S} \| \hat{\mathbf{x}}$ : For the magnetization pointing along the ribbon, there is no charge current $J^{0}$, and a spin polarization in both transverse directions is induced, indicating a spin torque according to Eq. (10). The $\sigma^{y}$ component is antisymmetric and, hence, constitutes a noncollinear magnetic order between the two edges. The $\sigma^{z}$ component is symmetric for the two edges, but has alternating signs along the ribbon, causing noncollinear magnetic order along the ribbon.

(iii) $\mathbf{S} \| \hat{\mathbf{y}}$ : For magnetization in-plane but perpendicular to the ribbon direction, an asymmetric band structure causes a nonchiral $J^{0}$, and the spin polarization in both transverse directions $\sigma^{x}$ and $\sigma^{z}$ are induced and are asymmetric between the two edges.

(iv) $\mathbf{S} \| \hat{\mathbf{z}}$ : The out-of-plane magnetization again causes a symmetric band structure. For the $+k_{x}$ state that is more localized at the right edge, we find a corresponding $-k_{x}$ state at the left edge, which suggests the existence of a chiral $J^{0}$. Interestingly, this feature is not obvious at low energy, but 


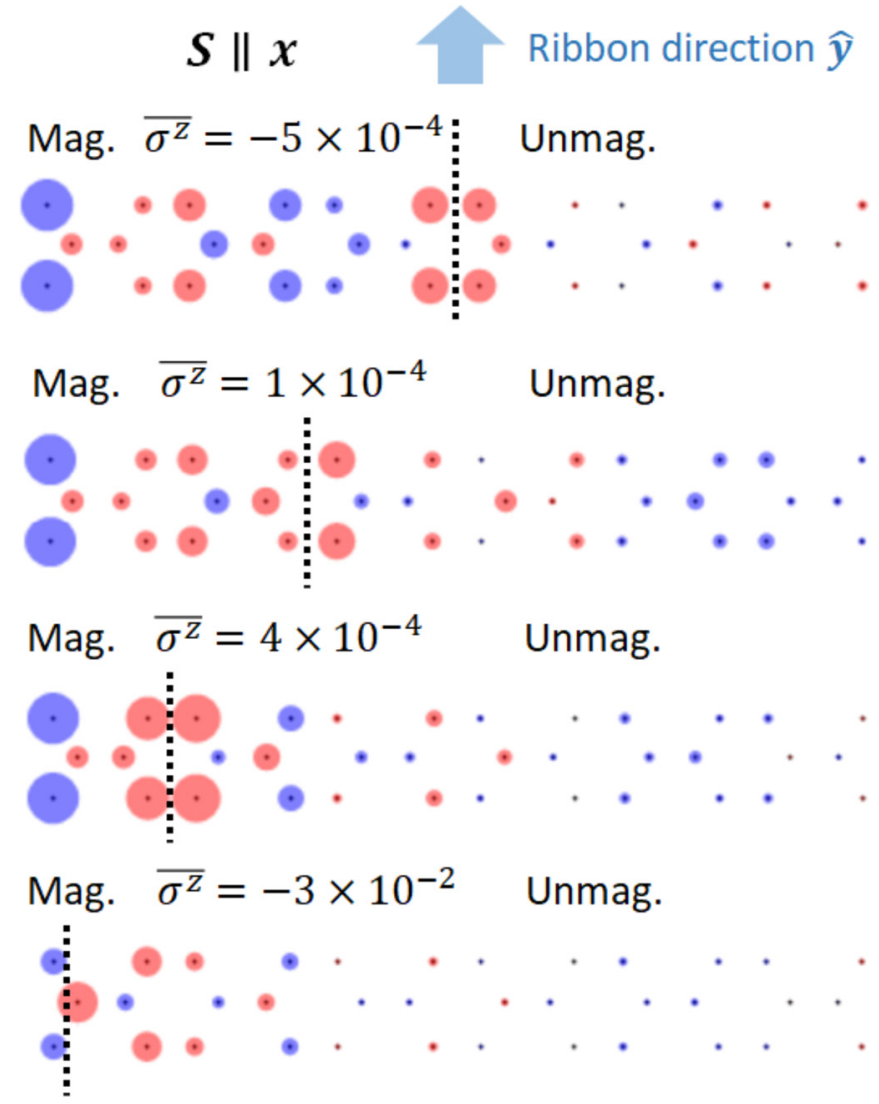

FIG. 4. The unit cell of partially magnetized zigzag ribbon at magnetization $\mathbf{S} \| \hat{\mathbf{x}}$, where the transverse spin polarization $\sigma^{z}$ is indicated by the size of disks, with red positive and blue negative. The region to the left of the dotted line is magnetized, and to the right is unmagnetized, and hence the panels from top to bottom represent the magnetization covering $1 / 2,1 / 3,1 / 6$, and the edge sites of the ribbon. The average spin polarization per site in the magnetized region is indicated by $\frac{\bar{\sigma}}{\sigma^{2}}$.

more prominent for the higher-energy states. Comparing this with the $\mathbf{S} \| \hat{\mathbf{y}}$ result, we again see that the chirality of $J^{0}$ can be controlled by the orientation of the magnetization. The $\sigma^{z}$ component is antisymmetric between the two edges, while $\sigma^{y}$ is symmetric but with sign-alternating along the ribbon. Finally, comparing the spin polarization $\sigma^{\alpha}$ in all three situations $\mathbf{S} \|\{\hat{\mathbf{x}}, \hat{\mathbf{y}}, \hat{\mathbf{z}}\}$, we conclude that every component of the local susceptibility tensor $\chi^{\alpha \beta}$ is nonzero, different from that of the zigzag ribbon in Sec. III B. Nevertheless, the total torque vanishes in all cases.

\section{Partially magnetized nanoribbons and irregular nanoflakes}

To make the magnetoelectric torque observable, one must overcome the overall canceling of the torque as seen in our discussion of the nanoribbons. We suggest two situations that the net torque can be nonzero. The first is to make the magnetization spatially varying, e.g., only occupy the region closer to one edge, such that the other edge is idle. Using a zigzag ribbon with $\mathbf{S} \| \hat{\mathbf{x}}$ as an example, in Fig. 4 we indeed see a nonzero net transverse spin polarization in this situation, whose average value per site is of the order of $\overline{\sigma^{z}} \sim 10^{-4}$

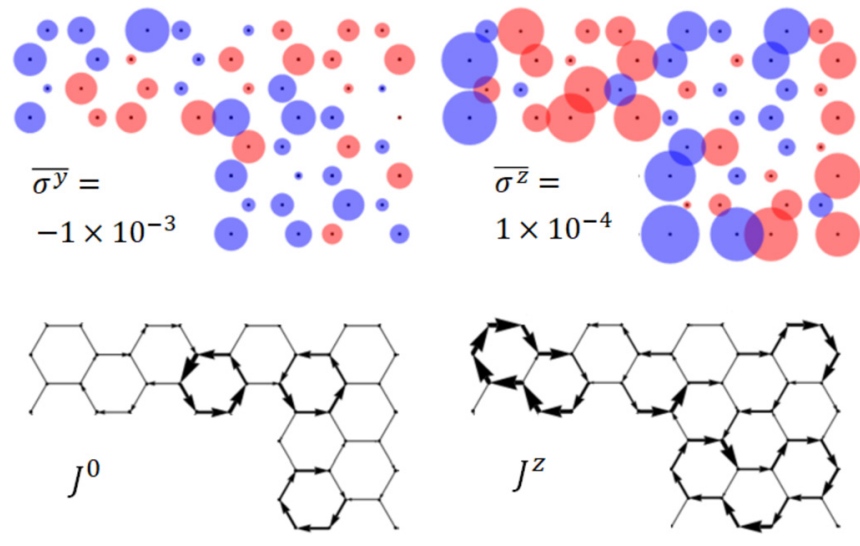

FIG. 5. An L-shaped nanoflake with magnetization $\mathbf{S} \| \hat{\mathbf{x}}$, where we show the transverse spin polarizations $\sigma^{y}$ and $\sigma^{z}$, charge current $J^{0}$, and out-of-plane polarized spin current $J^{z}$. The $\overline{\sigma^{y, z}}$ represents the average spin polarization per site.

when the magnetization only covers a region near the left edge, and it is dramatically enhanced to $\overline{\sigma^{z}} \sim 10^{-2}$ if the magnetization only exists on the edge sites. Following Eq. (10), the $\overline{\sigma^{z}} \sim 10^{-4}$ and $J_{\text {ex }} \sim 0.1 \mathrm{eV}$ would yield a very large spin torque $d \mathbf{S} / d t \sim 10 \mathrm{GHz}$. Although we likely overestimated this torque due to the large $J_{\mathrm{ex}}$ and $\lambda_{R}$ in Eq. (5), even if the torque is reduced by two orders of magnitude to $\sim 0.1$ $\mathrm{GHz}$, it is still significant.

The second proposal is to use graphene nanoflakes with irregular shapes, which may help to generate nonuniform distribution of spin polarization that does not sum to zero [87-91], given that the two edges are not equivalent or it may even be ambiguous to identify two opposite edges. In the example shown in Fig. 5, we see that the L-shaped nanoflake indeed yields a net $\overline{\sigma^{y}}$ and $\overline{\sigma^{z}}$ per site of the order of $10^{-3}$ to $10^{-4}$ when magnetization points at $\mathbf{S} \| \hat{\mathbf{x}}$, indicating a net torque of significant strength. In addition, despite the open boundary in all directions, still equilibrium currents $\left\{J^{0}, J^{x}, J^{y}, J^{z}\right\}$ exist. These currents turn into networks of local currents, suggesting that they survive even in realistic experimental situations of small and open boundary nanoflakes.

\section{CONCLUSIONS}

In summary, we demonstrate that graphene nanoribbons due to RSOC and geometric confinement display a peculiar magnetic response, including a bias-voltage-free spin torque, chiral and nonchiral edge currents, and equilibrium spin currents. Using a two-site toy model, we could analytically show features such as a transverse susceptibility caused by geometric confinement and linear in the RSOC strength. Numerical calculation suggests that the same features occur in nanoribbons. Given the controllability of RSOC by gate voltage [44-46,48-50], the transverse susceptibility can serve as a gate-voltage-induced magnetoelectric torque distinct from the usual current-induced spin-orbit torque. In a zigzag ribbon, this torque tends to create noncollinear spin polarization between the two edges, and in an armchair ribbon the noncollinear order is not only between the edges but can also be along the ribbon. Although the net torque sums to zero 
in uniformly magnetized ribbons, it can be nonzero if the magnetization only covers parts of the ribbon, say, closer to one edge, or in nanoflakes of irregular shapes. We estimate a net torque that can reach sub-GHz magnitude, pointing to the possibility of practical applications.

We further confirm that the equilibrium edge charge current $J^{0}$ predicted for $2 \mathrm{DEGs}$, when the magnetization points perpendicular to the edge, also exists in Rashba nanoribbons. Moreover, we discovered that $J^{0}$ can be nonchiral due to the asymmetric band structure, or chiral due to counterpropagating eigenstates localized at opposite edges, depending on the magnetization pointing in-plane or out-of-plane. In addition, the in-plane polarized persistent spin currents $J^{x}$ and $J^{y}$ that exist ubiquitously in 2D Rashba systems also manifest in nanoribbons, but the component flowing in the confined direction is strongly suppressed. Besides, the RSOC causes an out-of-plane polarized helical edge spin current $J^{z}$ even in the absence of magnetization. This discovery poses a challenge to distinguish $J^{z}$ from that of purely topological origin in graphene-based topological insulators containing RSOC, such as the Kane-Mele model [64,65,92]. Generally, the energy gap does not affect the existence of these currents and spin torques, since they are contributed from all the eigenstates in the Fermi sea, not only the low-energy flat band edge states that in some cases become dispersive. In addition, all these currents survive even in nanoflakes that have an open boundary in every direction. We anticipate that the controllability of RSOC by gate voltage and the magnetization by magnetic field offers a practical way to engineer these effects, which may help to realize them for practical purposes, such as building graphene-based spintronic devices [93-95].

\section{ACKNOWLEDGMENTS}

We thank J. C. Egues and A. Zegarra for fruitful discussions. This work is financially supported by a Productivity in Research Fellowship from CNPq.

\section{APPENDIX: EXPLICIT FORM OF THE CHARGE AND SPIN CURRENT OPERATORS}

To calculate the commutators in Eq. (6), we observe that the hopping and the Rashba Hamiltonians take the following general form,

$$
H_{t}+H_{R}=\sum_{i \in A} \sum_{\eta} \sum_{\alpha \beta}\left\{T_{\alpha \beta}^{\eta} c_{i \alpha}^{\dagger} c_{i+\eta \beta}+T_{\alpha \beta}^{\eta *} c_{i+\eta \beta}^{\dagger} c_{i \alpha}\right\},
$$

where $T_{\alpha \beta}^{\eta}$ is the hopping amplitude that the $\operatorname{spin} \beta$ at site $i+\eta$ becomes spin $\alpha$ at site $i$, and $T_{\alpha \beta}^{\eta *}$ is the complex conjugate of this amplitude. The results of the commutators give

$$
\begin{aligned}
J_{i, i+\eta}^{0}= & \frac{i a}{\hbar} \sum_{\alpha \beta}\left\{T_{\alpha \beta}^{\eta} c_{i \alpha}^{\dagger} c_{i+\eta \beta}-T_{\alpha \beta}^{\eta *} c_{i+\eta \beta}^{\dagger} c_{i \alpha}\right\}, \\
J_{i, i+\eta}^{a}= & \frac{i a}{\hbar} \sum_{\alpha \beta} \sum_{\mu \nu}\left\{T_{\alpha \beta}^{\eta} \delta_{\alpha \nu} \sigma_{\mu \nu}^{a} c_{i \mu}^{\dagger} c_{i+\eta \beta}\right. \\
& \left.-T_{\alpha \beta}^{\eta *} \delta_{\mu \alpha} \sigma_{\mu \nu}^{a} c_{i+\eta \beta}^{\dagger} c_{i \nu}\right\} .
\end{aligned}
$$

For $\boldsymbol{\eta}=\left(-\boldsymbol{\delta}_{1},-\boldsymbol{\delta}_{2},-\boldsymbol{\delta}_{3}\right)$, the complete list of nonzero hopping amplitudes are

$$
\begin{gathered}
T_{\uparrow \uparrow}^{-\delta_{i}}=T_{\downarrow \downarrow}^{-\delta_{i}}=t \quad \text { for } i=\{1,2,3\}, \\
T_{\uparrow \downarrow}^{-\delta_{1}}=i \lambda_{R}\left(-\frac{\sqrt{3}}{2}-\frac{i}{2}\right), \quad T_{\downarrow \uparrow}^{-\delta_{1}}=i \lambda_{R}\left(-\frac{\sqrt{3}}{2}+\frac{i}{2}\right), \\
T_{\uparrow \downarrow}^{-\delta_{2}}=i \lambda_{R}\left(\frac{\sqrt{3}}{2}-\frac{i}{2}\right), \quad T_{\downarrow \uparrow}^{-\delta_{2}}=i \lambda_{R}\left(\frac{\sqrt{3}}{2}+\frac{i}{2}\right), \\
T_{\uparrow \downarrow}^{-\delta_{3}}=-\lambda_{R}, \quad T_{\downarrow \uparrow}^{-\delta_{3}}=\lambda_{R},
\end{gathered}
$$

and $T_{\alpha \beta}^{\delta_{i}}=-T_{\alpha \beta}^{-\delta_{i}}$ for $\boldsymbol{\eta}=\left(\boldsymbol{\delta}_{1}, \boldsymbol{\delta}_{2}, \boldsymbol{\delta}_{3}\right)$. The explicit forms of the current operators are then given by

$$
\begin{aligned}
J_{i, i+\eta}^{0}= & \frac{i a}{\hbar}\left\{t c_{i \uparrow}^{\dagger} c_{i+\eta \uparrow}-t c_{i+\eta \uparrow}^{\dagger} c_{i \uparrow}+t c_{i \downarrow}^{\dagger} c_{i+\eta \downarrow}-t c_{i+\eta \downarrow}^{\dagger} c_{i \downarrow}\right. \\
& +T_{\uparrow \downarrow}^{\eta} c_{i \uparrow}^{\dagger} c_{i+\eta \downarrow}-T_{\uparrow \downarrow}^{\eta *} c_{i+\eta \downarrow}^{\dagger} c_{i \uparrow} \\
& \left.+T_{\downarrow \uparrow}^{\eta} c_{i \downarrow}^{\dagger} c_{i+\eta \uparrow}-T_{\downarrow \uparrow}^{\eta *} c_{i+\eta \uparrow}^{\dagger} c_{i \downarrow}\right\}, \\
J_{i, i+\eta}^{x}= & \frac{i a}{\hbar}\left\{t c_{i \downarrow}^{\dagger} c_{i+\eta \uparrow}-t c_{i+\eta \uparrow}^{\dagger} c_{i \downarrow}+t c_{i \uparrow}^{\dagger} c_{i+\eta \downarrow}-t c_{i+\eta \downarrow}^{\dagger} c_{i \uparrow}\right. \\
& +T_{\uparrow \downarrow}^{\eta} c_{i \downarrow}^{\dagger} c_{i+\eta \downarrow}-T_{\uparrow \downarrow}^{\eta *} c_{i+\eta \downarrow}^{\dagger} c_{i \downarrow} \\
& \left.+T_{\downarrow \uparrow}^{\eta} c_{i \uparrow}^{\dagger} c_{i+\eta \uparrow}-T_{\downarrow \uparrow}^{\eta *} c_{i+\eta \uparrow}^{\dagger} c_{i \uparrow}\right\}, \\
J_{i, i+\eta}^{y}= & \frac{a}{\hbar}\left\{-t c_{i \downarrow}^{\dagger} c_{i+\eta \uparrow}-t c_{i+\eta \uparrow}^{\dagger} c_{i \downarrow}+t c_{i \uparrow}^{\dagger} c_{i+\eta \downarrow}+t c_{i+\eta \downarrow}^{\dagger} c_{i \uparrow}\right. \\
& -T_{\uparrow \downarrow}^{\eta} c_{i \downarrow}^{\dagger} c_{i+\eta \downarrow}-T_{\uparrow \downarrow}^{\eta *} c_{i+\eta \downarrow}^{\dagger} c_{i \downarrow} \\
& \left.+T_{\downarrow \uparrow}^{\eta} c_{i \uparrow}^{\dagger} c_{i+\eta \uparrow}+T_{\downarrow \uparrow}^{\eta *} c_{i+\eta \uparrow}^{\dagger} c_{i \uparrow}\right\}, \\
& \left.+T_{\downarrow \uparrow}^{\eta} c_{i \downarrow}^{\dagger} c_{i+\eta \uparrow}+T_{\downarrow \uparrow}^{\eta *} c_{i+\eta \uparrow}^{\dagger} c_{i \downarrow}\right\} . \\
J_{i, i+\eta}^{z}= & \frac{i a}{\hbar}\left\{t c_{i \downarrow}^{\dagger} c_{i+\eta \uparrow}-t c_{i+\eta \uparrow}^{\dagger} c_{i \downarrow}-t c_{i \uparrow}^{\dagger} c_{i+\eta \downarrow}+t c_{i+\eta \downarrow}^{\dagger} c_{i \uparrow}\right. \\
& (\mathrm{A} 4) \\
&
\end{aligned}
$$

We then evaluate the equilibrium charge and spin current numerically by their expectation values

$$
\left\langle J_{i, i+\eta}^{a}\right\rangle=\sum_{n}\left\langle n\left|J_{i, i+\eta}^{a}\right| n\right\rangle f\left(E_{n}\right),
$$

where $|n\rangle$ is the eigenstate of the lattice Hamiltonian, and $f\left(E_{n}\right)=\left(e^{E_{n} / k_{B} T}+1\right)^{-1}$ is the Fermi distribution function. Note that this strategy includes all the eigenstates in the Fermi sea, in contrast to previous theories that consider only the low-energy states $[96,97]$. Often times we ignore the bracket $\langle\hat{\mathcal{O}}\rangle \equiv \hat{\mathcal{O}}$ of the expectation value for convenience. 
[1] Y. A. Bychkov and E. I. Rashba, Properties of a 2d electron gas with lifted spectral degeneracy, Pis'ma Zh. Eksp. Teor. Fiz. 39, 66 (1984) [JETP Lett. 39, 78 (1984)].

[2] Y. A. Bychkov and E. I. Rashba, Oscillatory effects and the magnetic susceptibility of carriers in inversion layers, J. Phys. C: Solid State Phys. 17, 6039 (1984).

[3] V. M. Edelstein, Spin polarization of conduction electrons induced by electric current in two-dimensional asymmetric electron systems, Solid State Commun. 73, 233 (1990).

[4] A. Manchon and S. Zhang, Theory of nonequilibrium intrinsic spin torque in a single nanomagnet, Phys. Rev. B 78, 212405 (2008).

[5] A. Manchon and S. Zhang, Theory of spin torque due to spinorbit coupling, Phys. Rev. B 79, 094422 (2009).

[6] P. M. Haney and M. D. Stiles, Current-Induced Torques in the Presence of Spin-Orbit Coupling, Phys. Rev. Lett. 105, 126602 (2010).

[7] P. Gambardella and I. M. Miron, Current-induced spinorbit torques, Phil. Trans. R. Soc. London A 369, 3175 (2011).

[8] D. A. Pesin and A. H. MacDonald, Quantum kinetic theory of current-induced torques in Rashba ferromagnets, Phys. Rev. B 86, 014416 (2012).

[9] P. M. Haney, H.-W. Lee, K.-J. Lee, A. Manchon, and M. D. Stiles, Current-induced torques and interfacial spin-orbit coupling, Phys. Rev. B 88, 214417 (2013).

[10] I. M. Miron, G. Gaudin, S. Auffret, B. Rodmacq, A. Schuhl, S. Pizzini, J. Vogel, and P. Gambardella, Current-driven spin torque induced by the Rashba effect in a ferromagnetic metal layer, Nat. Mater. 9, 230 (2010).

[11] I. M. Miron, K. Garello, G. Gaudin, P.-J. Zermatten, M. V. Costache, S. Auffret, S. Bandiera, B. Rodmacq, A. Schuhl, and P. Gambardella, Perpendicular switching of a single ferromagnetic layer induced by in-plane current injection, Nature (London) 476, 189 (2011).

[12] A. Manchon, J. Železný, I. M. Miron, T. Jungwirth, J. Sinova, A. Thiaville, K. Garello, and P. Gambardella, Current-induced spin-orbit torques in ferromagnetic and antiferromagnetic systems, Rev. Mod. Phys. 91, 035004 (2019).

[13] E. I. Rashba, Spin currents in thermodynamic equilibrium: The challenge of discerning transport currents, Phys. Rev. B 68, 241315(R) (2003).

[14] E. B. Sonin, Equilibrium spin currents in the Rashba medium, Phys. Rev. B 76, 033306 (2007).

[15] I. V. Tokatly, Equilibrium Spin Currents: Non-Abelian Gauge Invariance and Color Diamagnetism in Condensed Matter, Phys. Rev. Lett. 101, 106601 (2008).

[16] A. Reynoso, G. Usaj, M. J. Sánchez, and C. A. Balseiro, Theory of edge states in systems with Rashba spin-orbit coupling, Phys. Rev. B 70, 235344 (2004).

[17] V. L. Grigoryan, A. Matos Abiague, and S. M. Badalyan, Spin edge states: An exact solution and oscillations of the spin current, Phys. Rev. B 80, 165320 (2009).

[18] G. Usaj and C. A. Balseiro, Spin accumulation and equilibrium currents at the edge of 2DEGs with spin-orbit coupling, Europhys. Lett. 72, 631 (2005).

[19] A. Zegarra, J. C. Egues, and W. Chen, Persistent currents and spin torque caused by percolated quantum spin Hall state, Phys. Rev. B 101, 224438 (2020).
[20] W. Chen, Spin torque and persistent currents caused by percolation of topological surface states, Phys. Rev. B 102, 144442 (2020).

[21] K. S. Novoselov, A. K. Geim, S. V. Morozov, D. Jiang, M. I. Katsnelson, I. V. Grigorieva, S. V. Dubonos, and A. A. Firsov, Two-dimensional gas of massless Dirac fermions in graphene, Nature (London) 438, 197 (2005).

[22] S. Y. Zhou, G.-H. Gweon, J. Graf, A. V. Fedorov, C. D. Spataru, R. D. Diehl, Y. Kopelevich, D.-H. Lee, S. G. Louie, and A. Lanzara, First direct observation of Dirac fermions in graphite, Nat. Phys. 2, 595 (2006).

[23] A. K. Geim and K. S. Novoselov, The rise of graphene, Nat. Mater. 6, 183 (2007).

[24] A. H. Castro Neto, F. Guinea, N. M. R. Peres, K. S. Novoselov, and A. K. Geim, The electronic properties of graphene, Rev. Mod. Phys. 81, 109 (2009).

[25] A. K. Geim, Graphene: Status and prospects, Science 324, 1530 (2009).

[26] S. Das Sarma, S. Adam, E. H. Hwang, and E. Rossi, Electronic transport in two-dimensional graphene, Rev. Mod. Phys. 83, 407 (2011).

[27] M. Fujita, K. Wakabayashi, K. Nakada, and K. Kusakabe, Peculiar localized state at zigzag graphite edge, J. Phys. Soc. Jpn. 65, 1920 (1996).

[28] K. Nakada, M. Fujita, G. Dresselhaus, and M. S. Dresselhaus, Edge state in graphene ribbons: Nanometer size effect and edge shape dependence, Phys. Rev. B 54, 17954 (1996).

[29] L. Brey and H. A. Fertig, Electronic states of graphene nanoribbons studied with the Dirac equation, Phys. Rev. B 73, 235411 (2006).

[30] M. Ezawa, Peculiar width dependence of the electronic properties of carbon nanoribbons, Phys. Rev. B 73, 045432 (2006).

[31] N. M. R. Peres, F. Guinea, and A. H. Castro Neto, Electronic properties of disordered two-dimensional carbon, Phys. Rev. B 73, 125411 (2006).

[32] A. R. Akhmerov and C. W. J. Beenakker, Boundary conditions for Dirac fermions on a terminated honeycomb lattice, Phys. Rev. B 77, 085423 (2008).

[33] C. Tao, L. Jiao, O. V. Yazyev, Y.-C. Chen, J. Feng, X. Zhang, R. B. Capaz, J. M. Tour, A. Zettl, S. G. Louie, H. Dai, and M. F. Crommie, Spatially resolving edge states of chiral graphene nanoribbons, Nat. Phys. 7, 616 (2011).

[34] P. Ruffieux, S. Wang, B. Yang, C. Sánchez-Sánchez, J. Liu, T. Dienel, L. Talirz, P. Shinde, C. A. Pignedoli, D. Passerone, T. Dumslaff, X. Feng, K. Müllen, and R. Fasel, On-surface synthesis of graphene nanoribbons with zigzag edge topology, Nature (London) 531, 489 (2011).

[35] Y.-W. Son, M. L. Cohen, and S. G. Louie, Energy Gaps in Graphene Nanoribbons, Phys. Rev. Lett. 97, 216803 (2006).

[36] Y.-W. Son, M. L. Cohen, and S. G. Louie, Half-metallic graphene nanoribbons, Nature (London) 444, 347 (2006).

[37] V. Barone, O. Hod, and G. E. Scuseria, Electronic structure and stability of semiconducting graphene nanoribbons, Nano Lett. 6, 2748 (2006).

[38] L. Yang, C.-H. Park, Y.-W. Son, M. L. Cohen, and S. G. Louie, Quasiparticle Energies and Band Gaps in Graphene Nanoribbons, Phys. Rev. Lett. 99, 186801 (2007).

[39] M. Y. Han, B. Özyilmaz, Y. Zhang, and P. Kim, Energy BandGap Engineering of Graphene Nanoribbons, Phys. Rev. Lett. 98, 206805 (2007). 
[40] K. A. Ritter and J. W. Lyding, The influence of edge structure on the electronic properties of graphene quantum dots and nanoribbons, Nat. Mater. 8, 235 (2009).

[41] K. Wakabayashi, M. Fujita, H. Ajiki, and M. Sigrist, Electronic and magnetic properties of nanographite ribbons, Phys. Rev. B 59, 8271 (1999).

[42] H. Lee, Y.-W. Son, N. Park, S. Han, and J. Yu, Magnetic ordering at the edges of graphitic fragments: Magnetic tail interactions between the edge-localized states, Phys. Rev. B 72, 174431 (2005).

[43] L. Pisani, J. A. Chan, B. Montanari, and N. M. Harrison, Electronic structure and magnetic properties of graphitic ribbons, Phys. Rev. B 75, 064418 (2007).

[44] B. Yang, M.-F. Tu, J. Kim, Y. Wu, H. Wang, J. Alicea, R. $\mathrm{Wu}, \mathrm{M}$. Bockrath, and J. Shi, Tunable spin-orbit coupling and symmetry-protected edge states in graphene/ $\mathrm{WS}_{2}, 2 \mathrm{D}$ Mater. 3, 031012 (2016).

[45] Z. Wang, D.-K. Ki, J. Y. Khoo, D. Mauro, H. Berger, L. S. Levitov, and A. F. Morpurgo, Origin and Magnitude of "Designer" Spin-Orbit Interaction in Graphene on Semiconducting Transition Metal Dichalcogenides, Phys. Rev. X 6, 041020 (2016).

[46] B. Yang, M. Lohmann, D. Barroso, I. Liao, Z. Lin, Y. Liu, L. Bartels, K. Watanabe, T. Taniguchi, and J. Shi, Strong electron-hole symmetric Rashba spin-orbit coupling in graphene/monolayer transition metal dichalcogenide heterostructures, Phys. Rev. B 96, 041409(R) (2017).

[47] M. Offidani, M. Milletarì, R. Raimondi, and A. Ferreira, Optimal Charge-to-Spin Conversion in Graphene on TransitionMetal Dichalcogenides, Phys. Rev. Lett. 119, 196801 (2017).

[48] C. K. Safeer, J. Ingla-Aynés, F. Herling, J. H. Garcia, M. Vila, N. Ontoso, M. Reyes Calvo, S. Roche, L. E. Hueso, and F. Casanova, Room-temperature spin Hall effect in graphene $/ \mathrm{MoS}_{2}$ van der Waals Heterostructures, Nano Lett. 19, 1074 (2019).

[49] T. S. Ghiasi, A. A. Kaverzin, P. J. Blah, and B. J. van Wees, Charge-to-spin conversion by the Rashba-Edelstein effect in two-dimensional van der Waals heterostructures up to room temperature, Nano Lett. 19, 5959 (2019).

[50] L. A. Benítez, W. Savero Torres, J. F. Sierra, M. Timmermans, J. H. Garcia, S. Roche, M. V. Costache, and S. O. Valenzuela, Tunable room-temperature spin galvanic and spin Hall effects in van der Waals heterostructures, Nat. Mater. 19, 170 (2020).

[51] J. B. S. Mendes, O. Alves Santos, L. M. Meireles, R. G. Lacerda, L. H. Vilela-Leão, F. L. A. Machado, R. L. RodríguezSuárez, A. Azevedo, and S. M. Rezende, Spin-Current to Charge-Current Conversion and Magnetoresistance in a Hybrid Structure of Graphene and Yttrium Iron Garnet, Phys. Rev. Lett. 115, 226601 (2015).

[52] Z. Wang, C. Tang, R. Sachs, Y. Barlas, and J. Shi, ProximityInduced Ferromagnetism in Graphene Revealed by the Anomalous Hall Effect, Phys. Rev. Lett. 114, 016603 (2015).

[53] S. Dushenko, H. Ago, K. Kawahara, T. Tsuda, S. Kuwabata, T. Takenobu, T. Shinjo, Y. Ando, and M. Shiraishi, Gate-Tunable Spin-Charge Conversion and the Role of Spin-Orbit Interaction in Graphene, Phys. Rev. Lett. 116, 166102 (2016).

[54] J. C. Leutenantsmeyer, A. A. Kaverzin, M. Wojtaszek, and B. J. van Wees, Proximity induced room temperature ferro- magnetism in graphene probed with spin currents, 2D Mater. 4, 014001 (2016).

[55] A. G. Rybkin, A. A. Rybkina, M. M. Otrokov, O. Y. Vilkov, I. I. Klimovskikh, A. E. Petukhov, M. V. Filianina, V. Y. Voroshnin, I. P. Rusinov, A. Ernst, A. Arnau, E. V. Chulkov, and A. M. Shikin, Magneto-spin-orbit graphene: Interplay between exchange and spin-orbit couplings, Nano Lett. 18, 1564 (2018).

[56] G. S. Diniz, M. R. Guassi, and F. Qu, Engineering the quantum anomalous Hall effect in graphene with uniaxial strains, J. Appl. Phys. 114, 243701 (2013).

[57] M. R. Guassi, G. S. Diniz, N. Sandler, and F. Qu, Zero-field and time-reserval-symmetry-broken topological phase transitions in graphene, Phys. Rev. B 92, 075426 (2015).

[58] M. Offidani and A. Ferreira, Anomalous Hall Effect in 2D Dirac Materials, Phys. Rev. Lett. 121, 126802 (2018).

[59] A. Dyrdał and J. Barnaś, Current-induced spin polarization and spin-orbit torque in graphene, Phys. Rev. B 92, 165404 (2015).

[60] H. Li and A. Manchon, Tunable spin-charge conversion through topological phase transitions in zigzag nanoribbons, Phys. Rev. B 93, 235317 (2016).

[61] M. Rodriguez-Vega, G. Schwiete, J. Sinova, and E. Rossi, Giant Edelstein effect in topological-insulator-graphene heterostructures, Phys. Rev. B 96, 235419 (2017).

[62] K. Zollner, M. D. Petrović, K. Dolui, P. Plecháč, B. K. Nikolić, and J. Fabian, Scattering-induced and highly tunable by gate damping-like spin-orbit torque in graphene doubly proximitized by two-dimensional magnet $\mathrm{Cr}_{2} \mathrm{Ge}_{2} \mathrm{Te}_{6}$ and monolayer $\mathrm{WS}_{2}$, Phys. Rev. Res. 2, 043057 (2020).

[63] A. A. Rybkina, A. G. Rybkin, I. I. Klimovskikh, P. N. Skirdkov, K. A. Zvezdin, A. K. Zvezdin, and A. M. Shikin, Advanced graphene recording device for spin-orbit torque magnetoresistive random access memory, Nanotechnology 31, 165201 (2020).

[64] C. L. Kane and E. J. Mele, Quantum Spin Hall Effect in Graphene, Phys. Rev. Lett. 95, 226801 (2005).

[65] C. L. Kane and E. J. Mele, $Z_{2}$ Topological Order and the Quantum Spin Hall Effect, Phys. Rev. Lett. 95, 146802 (2005).

[66] H. Min, J. E. Hill, N. A. Sinitsyn, B. R. Sahu, L. Kleinman, and A. H. MacDonald, Intrinsic and Rashba spin-orbit interactions in graphene sheets, Phys. Rev. B 74, 165310 (2006).

[67] A. Dyrdał and J. Barnaś, Anomalous, spin, and valley Hall effects in graphene deposited on ferromagnetic substrates, 2D Mater. 4, 034003 (2017).

[68] M. Peralta, E. Medina, and F. Mireles, Proximity-induced exchange and spin-orbit effects in graphene on Ni and Co, Phys. Rev. B 99, 195452 (2019).

[69] H. Katsura, N. Nagaosa, and A. V. Balatsky, Spin Current and Magnetoelectric Effect in Noncollinear Magnets, Phys. Rev. Lett. 95, 057205 (2005).

[70] P. Bruno and V. K. Dugaev, Equilibrium spin currents and the magnetoelectric effect in magnetic nanostructures, Phys. Rev. B 72, 241302(R) (2005).

[71] W. Chen, P. Horsch, and D. Manske, Dissipationless spin current between two coupled ferromagnets, Phys. Rev. B 89, 064427 (2014).

[72] I. Dzyaloshinsky, A thermodynamic theory of "weak" ferromagnetism of antiferromagnetics, J. Phys. Chem. Solids 4, 241 (1958). 
[73] T. Moriya, Anisotropic superexchange interaction and weak ferromagnetism, Phys. Rev. 120, 91 (1960).

[74] Q.-F. Sun and X. C. Xie, Persistent spin current in spin-orbit coupling systems in the absence of an external magnetic field, Int. J. Mod. Phys. B 21, 3687 (2007).

[75] E. Nakhmedov and O. Alekperov, Out-of-plane equilibrium spin current in a quasi-two-dimensional electron gas under inplane magnetic field, Phys. Rev. B 85, 153302 (2012).

[76] W. Chen, Absence of equilibrium edge currents in theoretical models of topological insulators, Phys. Rev. B 101, 195120 (2020).

[77] W. Chen and A. P. Schnyder, Majorana edge states in superconductor-noncollinear magnet interfaces, Phys. Rev. B 92, 214502 (2015).

[78] K. Nomura, J. Wunderlich, J. Sinova, B. Kaestner, A. H. MacDonald, and T. Jungwirth, Edge-spin accumulation in semiconductor two-dimensional hole gases, Phys. Rev. B 72, 245330 (2005).

[79] P. Bokes and F. Horváth, Edge-induced spin polarization in twodimensional electron gas, Phys. Rev. B 81, 125302 (2010).

[80] S. I. Erlingsson, J. Carlos Egues, and D. Loss, Spin densities in parabolic quantum wires with Rashba spin-orbit interaction, Phys. Status Solidi C 3, 4317 (2007).

[81] W. Zhang, Voltage-driven spintronic logic gates in graphene nanoribbons, Sci. Rep. 4, 6320 (2014).

[82] A. Khaetskii, Edge spin accumulation in two-dimensional electron and hole systems in a quasiballistic regime, Phys. Rev. B 89, 195408 (2014).

[83] A. Khaetskii and J. C. Egues, Giant edge spin accumulation in a symmetric quantum well with two subbands, Europhys. Lett. 118, 57006 (2017).

[84] F. Sousa, G. Tatara, and A. Ferreira, Skew-scatteringinduced giant antidamping spin-orbit torques: Collinear and out-of-plane Edelstein effects at two-dimensional material/ferromagnet interfaces, Phys. Rev. Res. 2, 043401 (2020).

[85] T. P. Cysne, F. S. M. Guimarães, L. M. Canonico, T. G. Rappoport, and R. B. Muniz, Orbital magnetoelectric effect in zigzag nanoribbons of $p$-band systems, arXiv:2101.05049.
[86] J. Klinovaja, P. Stano, A. Yazdani, and D. Loss, Topological Superconductivity and Majorana Fermions in RKKY Systems, Phys. Rev. Lett. 111, 186805 (2013).

[87] Z. Ma and W. Sheng, A spin-valve device based on dumbbellshaped graphene nanoislands, Appl. Phys. Lett. 99, 083101 (2011).

[88] I. Weymann, J. Barnaś, and S. Krompiewski, Manifestation of the shape and edge effects in spin-resolved transport through graphene quantum dots, Phys. Rev. B 85, 205306 (2012).

[89] A. D. Güçlü, P. Potasz, and P. Hawrylak, Graphene-based integrated electronic, photonic and spintronic circuit, in Future Trends in Microelectronics (John Wiley and Sons, 2013), pp. 308-318.

[90] K. Luo and W. Sheng, Many-body effects in the spin-polarized electron transport through graphene nanoislands, J. Appl. Phys. 115, 053705 (2014).

[91] K. Szałowski, Graphene nanoflakes in external electric and magnetic in-plane fields, J. Magn. Magn. Mater. 382, 318 (2015).

[92] L. R. F. Lima and C. Lewenkopf, RKKY interaction in carbon nanotubes and graphene nanoribbons, arXiv:2104.07206.

[93] D. Pesin and A. H. MacDonald, Spintronics and pseudospintronics in graphene and topological insulators, Nat. Mater. 11, 409 (2012).

[94] W. Han, R. K. Kawakami, M. Gmitra, and J. Fabian, Graphene spintronics, Nat. Nanotechnol. 9, 794 (2014).

[95] S. Roche, J. Åkerman, B. Beschoten, J.-C. Charlier, M. Chshiev, S. P. Dash, B. Dlubak, J. Fabian, A. Fert, M. Guimarães, F. Guinea, I. Grigorieva, C. Schönenberger, P. Seneor, C. Stampfer, S. O. Valenzuela, X. Waintal, and B. van Wees, Graphene spintronics: The European flagship perspective, 2D Mater. 2, 030202 (2015).

[96] H. Zhang, Z. Ma, and J.-F. Liu, Equilibrium spin current in graphene with Rashba spin-orbit coupling, Sci. Rep. 4, 6464 (2014).

[97] Z.-X. Li and H. Yao, Edge stability and edge quantum criticality in two-dimensional interacting topological insulators, Phys. Rev. B 96, 241101(R) (2017). 